\title{
Toward Comprehensive Clinical Care for a Complex Condition: Introduction to the Special Issue on Tourette Syndrome
}

\author{
Michael B. Himle • Douglas W. Woods
}

Published online: 28 January 2011

(C) Springer Science+Business Media, LLC 2011

Tourette's Syndrome (TS) is a complex neurobiological disorder that typically begins in early childhood. Once thought to be extremely rare, recent estimates have shown it to be more common in school-aged children than was previously thought (Scahill et al. 2005). Although the range in severity is considerable, TS is a significant source of impairment for many afflicted children and families (Storch et al. 2007; Woods and Himle 2005).

Over the course of the disorder, the involuntary tics that characterize TS change in presentation and fluctuate in topography, number, frequency, and complexity across days, weeks, and months (Leckman et al. 1998). In addition to tics, most children with TS struggle with one or more comorbid condition, most commonly attention deficit hyperactivity disorder (ADHD), obsessive-compulsive disorder (OCD), oppositional-defiant disorder (ODD), conduct disorder (CD), explosive outbursts ("rage attacks"), and/or a learning disorder (Freeman et al. 2007). The complex combination of tics, internalizing problems, and externalizing problems results in significant interpersonal, family, and academic impairment and creates challenges for effective clinical care.

The past two decades have witnessed a surge in empirical research aimed at enhancing clinical care for TS. Advances in genetic and brain imaging techniques have shed light on the underlying phathophysiology which has in turn led to the development and testing of more effective pharmacotherapeutics. Refined assessment techniques have allowed for reliable diagnosis, more precise prevalence estimates, and a better understanding of the TS phenotype. Psychosocial research has led to the development and testing of exciting new nonpharmacological treatments for tics and associated symptoms. This is perhaps best exemplified in the recently published Comprehensive Behavioral Treatment for Tics (CBIT) treatment trial

\footnotetext{
M. B. Himle $(\bowtie)$

Department of Psychology, University of Utah, 380 S 1530 E \#502, Salt Lake City, UT 84112, USA e-mail: Michael.himle@utah.edu

D. W. Woods

University of Wisconsin, Milwaukee, WI, USA
} 
(Piacentini et al. 2010). In the largest clinical trial with TS children to date $(N=126)$, the CBIT team compared a state-of-the-art behavioral intervention for tics (CBIT, consisting of psychoeducation, habit reversal training, function-based assessment and treatment, relaxation training, and a behavioral rewards program) to a psychoeducation + supportive psychotherapy control. Results showed that CBIT was more effective at reducing tic severity and tic-related impairment than the control treatment and that treatment gains were maintained at 6-months post-treatment

While advancements such as CBIT are encouraging, there are still considerable barriers to effective clinical care for TS. To start, many children do not get diagnosed at an early age, which delays treatment. Second, the clinical complexities of TS are not sufficiently understood, making clinical decision making difficult (e.g., deciding what aspects of which symptoms to address first and how). Third, although the field has made significant advances in treatment (both pharmacological and nonpharmacological), not all children respond to available interventions and many children derive only moderate benefit, emphasizing the need for continued treatment development and testing. Finally, many children do not have access to empirically supported treatments due to a variety of factors, one of which is a lack of trained providers. The articles in this special issue begin to address the barriers surrounding early identification, clinical assessment and diagnosis, treatment planning, treatment development and administration, and dissemination and utilization of empirically-supported assessment and treatment strategies.

In the first article, White et al. (2011 [this issue]) appropriately note that inadequate recognition of mental health problems is a major barrier to the provision of empirically-based clinical care. Although the clinical, research, and advocacy communities have made considerable strides in enhancing early identification of children with TS, many children still go unrecognized. One way to enhance early identification is through education. White et al. examined the effectiveness of a workshop designed to teach elementary school teachers about TS and related conditions. At noted by the authors, teachers are in a unique position that may allow them to identify children with TS, initiate clinical referral, and help inform practitioners of the child's clinical and academic needs.

The second article in the issue demonstrates the complexity of TS and emphasizes the need for future research to inform clinical decision-making. In this article, Specht and the rest of the CBIT trial team (Specht et al. 2011, [this issue]) report on the clinical characteristics of the CBIT sample, which is one of the largest clinical samples of TS children assessed using a comprehensive state-of-the-art battery. This study confirms the diagnostic complexity of TS and explores the degree to which various aspects of tic severity (including the number, frequency, complexity, intensity, and interference) and various comorbid conditions contribute to overall impairment in children and families with TS. The findings of this study are important because they provide practitioners guidance in assessment and intervention planning.

It is fitting that the Specht et al. article - showing high rates of comorbidity and associated impairment - is followed by Leclerc et al. (2011 [this issue]) who report on one of the first studies to examine a behavioral treatment program for explosive outbursts (EOs) in children with TS. Explosive outbursts, or "rage attacks," occur in a significant portion of children with TS. These episodes are characterized by sudden, unpredictable, and uncontrollable physical and/or verbal aggressions that are excessive given the individual's developmental level and the provocation (Budman 
et al. 2000). They are often targeted at family members or personal/family property, creating significant family strife as well as remorse and regret in the child (not to mention occasional harm to self or others). Despite the relatively high prevalence and the significant sequela that results from EOs, few studies have directly examined nonpharmacological treatment options. Leclerc et al. examined the efficacy of a behavioral program for managing EOs in two children with TS. In addition to outlining a novel strategy for addressing these symptoms, the study highlights difficulties likely to be faced by practitioners working with children with EOs.

Given that the issue began with the first step in comprehensive clinical care (i.e., identification, White et al.), it is fitting that it ends with two articles that aim to enhance treatment and improve treatment dissemination and utilization/provision. As noted above, there is considerable evidence demonstrating the efficacy of behavioral therapy for managing tics in children with TS. However, like any intervention, behavior therapy does not work for all children and rarely results in complete elimination of tics. In the fourth paper of this series, Franklin et al. (2011 [this issue]) developed and tested an acceptance-enhanced habit reversal training (HRT + ACT) protocol for delivering habit reversal training (HRT) and directly compared the enhanced treatment to HRT as usual. Although their study did not show that ACT + HRT significantly enhanced HRT as usual (both treatments were effective), it is yet another confirmation of the important role of behavior therapy in comprehensive clinical care for TS. Furthermore, the Franklin et al. (2011, [this issue]) study demonstrated that therapists with a CBT background (but little experience with TS) can be efficiently trained to deliver HRT effectively for TS. This is important because HRT has not been widely adopted in the professional community. For example, one recent survey of practitioners found that less than $10 \%$ reported knowing how to implement HRT. This is perhaps because very few therapists are trained in HRT (Marcks et al. 2004).

In the final article in the special series, Flancbaum et al. (2011, [this issue]) address another important issues in intervention and dissemination research, adapting the treatment schedule. In their article, they present a case study in which they adapted HRT for intensive delivery for an individual with TS who would otherwise not have had access to treatment. Manualized treatments are often adapted to meet the needs of individual clients or because it is believed that the patient requires an intensive "dose" of treatment. The Flacbaum et al. case study addresses and outlines the advantages and challenges to modifying HRT for intense delivery over a short period of time. Additional studies evaluating ways to modify empirically supported treatments such that treatment utilization is enhanced and effectiveness is preserved are sorely needed.

Combined, these studies represent the expansion of the field's understanding and approach to the clinical care of persons with Tourette syndrome. It is our hope that this series of papers can serve as a springboard for additional research on this important, but understudied condition.

\section{References}

Budman, C. L., Bruun, R. D., Park, K. S., Lesser, M., \& Olson, M. (2000). Explosive outbursts in children with

Tourette disorder. Journal of the American Academy of Child and Adolescent Psychiatry, 39, 1270-1276. 
Flancbaum, M., Rockmore, L., \& Franklin, M. E. (2011). Intensive behavior therapy for tics: Implications for clinical practice and overcoming barriers to treatment. Journal of Developmental and Physical Disabilities.

Franklin, M. E., Best, S. H., Wilson, M. A., Loew, B., \& Compton, S. N. (2011). Habit reversal training and acceptance and commitment therapy for Tourette syndrome: A pilot project. Journal of Developmental and Physical Disabilities.

Freeman, R. D., Fast, D. K., Burd, L., Kerbeshian, J., Robertson, M. M., \& Sandor, P. (2007). An international perspective on Tourette syndrome: selected findings from 3500 individuals in 22 countries. Developmental Medicine and Child Neurology, 42, 436-447.

Leckman, J. F., Zhang, H., Vitale, A., Lahnin, F., Lynch, K., Bondi, C., et al. (1998). Course of tic severity in Tourette syndrome: The first two decades. Pediatrics, 102, 14-19.

Leclerc, J., O'Connor, K. P., Forget, J., \& Lavioe, M. E. (2011). Behavioral program for managing explosive outbursts in children with Tourette syndrome. Journal of Developmental and Physical Disabilities.

Marcks, B. A., Woods, D. W., Teng, E. J., \& Twohig, M. P. (2004). What do those who know, know? Investigating providers' knowledge about Tourette syndrome and its treatment. Cognitive and Behavioral Practice, 11, 298-305.

Piacentini, J., Woods, D. W., Scahill, L. D., Wilhelm, S., Peterson, A., Chang, S., et al. (2010). Behavior Therapy for Children with Tourette Syndrome: A Randomized Controlled Trial. Journal of the American Medical Association, 303, 1929-1937.

Scahill, L., Sukhodolsky, D., Williams, S., \& Leckman, J. F. (2005). The public health importance of tics and tic disorders. Advances in Neurology, 96, 240-248.

Specht, M. W., Woods, D. W., Piacentini, J., Scahill, L., Wilhelm, S., Peterson, A. L.,...\& Walkup, J. T. (2011). Clinical characteristics of children and adolescents with a primary tic disorder. Journal of Developmental and Physical Disabilities.

Storch, E. A., Merlo, L. J., Lack, C., Milsom, V. A., Geffken, G. R., Goodman, W. K., et al. (2007). Quality of life in youth with Tourette's syndrome and chronic tic disorder. Journal of Clinical Child and Adolescent Psychology, 36, 217-227.

White, S. W., Sukhodolsky, D. G., Rains, A. L., Foster, D., McGuire, J. F., \& Scahill, L. (2011). Elementary school teachers' knowledge of Tourette syndrome, obsessive-compulsive disorder, \& attention-deficit/hyperactivity disorder: Effects of teacher training. Journal of Developmental and Physical Disabilities.

Woods, D. W., \& Himle, M. B. (2005). The use of the impact on family scale in children with Tourette's syndrome: Descriptive data and concurrent validity. Child and Family Behavior Therapy, 27, 11-21.

Drs. Himle and Woods would like to thank Dr. Michel Hersen for the opportunity to guest edit this special issue on Tourette syndrome. They would also like to thank those who served as reviewers for this issue, including Christopher Flessner, Martin Franklin, Rebecca Johnson, Kieron O'Connor, Jeff Sigafoos, Matt Specht, Eric Storch, Denis Sukhodolsky, and Chad Wetterneck. 